\title{
EL SÍNDROME DE BURNOUT EN UN GRUPO DE PROFESORES DE ENSEÑANZA SECUNDARIA EN MONTEVIDEO
}

\author{
BURNOUT SYNDROME IN A GROUP OF SECONDARY SCHOOL TEACHERS IN \\ MONTEVIDEO
}

\author{
Natalia Colino \\ Pablo Pérez de León \\ Universidad Católica del Uruguay
}

\begin{abstract}
Resumen: Este trabajo estudia la incidencia del síndrome de burnout en un grupo de profesores uruguayos. Una muestra de 279 docentes, quienes trabajan en centros de enseñanza públicos en Montevideo, completaron cuestionarios acerca de: síndrome de burnout, personalidad, eventos de vida, áreas de la vida laboral, intenciones de abandono y datos sociodemográficos. Los resultados obtenidos muestran que el 4,2\% de los docentes encuestados presentaron elevados niveles en el síndrome de burnout y un $42 \%$ obtuvieron altos valores en al menos una de sus dimensiones. El género femenino, los docentes con elevada cantidad de horas semanales de trabajo, con poca antigüedad en la profesión, que no poseen formación docente y que trabajan hasta en dos centros educativos, son quienes puntuaron más alto en la escala del síndrome de burnout. La congruencia entre diversas áreas de la vida laboral y los valores de los participantes, la personalidad tipo A, los eventos de vida estresantes y las intenciones de abandono correlacionaron significativamente con el síndrome de burnout.
\end{abstract}

Palabras Clave: síndrome de quemarse en el trabajo; profesores; personalidad tipo A; eventos de vida; intenciones de abandono

\begin{abstract}
This article examines the incidence of the burnout syndrome among Uruguayan teachers. A sample of 279 secondary school teachers, who work in public schools in Montevideo, completed questionnaires about: burnout syndrome, personality, life events, areas of working life, turnover intentions and sociodemographic data. The results show that $4.2 \%$ of the teachers surveyed had elevated levels of burnout syndrome and $42 \%$ obtained high values in at least one dimension of the syndrome. The characteristics displayed by the educators who scored higher on the scale of burnout syndrome are the following: the female gender, high number of weekly hours of work, little seniority in the profession, lack of educational training and work in up to two schools. The job-person fit, the type A behavior, stressful life events and turnover intentions correlated significantly with burnout syndrome.
\end{abstract}

Key Words: burnout syndrome; teachers; type A personality; life events; turnover intention

\section{Introducción}

Desde hace años, el colectivo docente ha estado sufriendo cambios en sus condiciones de trabajo: el deterioro salarial, el multiempleo, la sobrecarga laboral y la universalización de la enseñanza secundaria ${ }^{1}$ que no vino acompañada de un incremento suficiente en la construcción de centros educativos ni tampoco de una preparación a los docentes para las nuevas

\footnotetext{
1 En Uruguay, "la educación secundaria o media, está organizada en dos ciclos, cada uno con tres años de duración: el Ciclo Básico, obligatorio para el grupo de edad 12 a 14 años, que se imparte en la modalidad general y en la tecnológica (enseñanza técnica) y el ciclo superior (Bachillerato, grupo de edad 15 a 17 años), también obligatorio según la Ley de Educación de 2008" (United Nations Organization for Education, Science and Culture [UNESCO], 2010, p. 13).
}

poblaciones que llegarían a sus aulas. Al mismo tiempo, las familias se han ido desarticulado en forma alarmante, no pudiendo a veces cumplir con su rol socializador. Es de esta manera, que las aulas se han superpoblado, la indisciplina se ha acentuado y las tareas docentes se han ampliado cada vez más. A partir de esta realidad, se ha generado un desánimo generalizado en el colectivo docente, quien, además, ha recibido el cuestionamiento de la sociedad. (Cornejo, 2008; Robalino \& Körner, 2005; Rossi, 2009).

Una respuesta prolongada a la incidencia de estos y otros estresores laborales, emocionales e interpersonales determinan la presencia del síndrome de burnout (SB). 
EI SB está definido por tres dimensiones: agotamiento emocional, despersonalización y baja realización personal (Maslach, Schaufeli \& Leiter, 2001). Constituye uno de los riesgos psicosociales en el lugar de trabajo (GonzálezTrijueque, Giachero \& Delgado, 2012).

La investigación acerca del SB ha crecido en los pasados 40 años, atravesando distintas fases en su desarrollo. Maslach et al. (2001) han identificado la existencia de una primera fase exploratoria y una segunda fase empírica. La primera fase adquirió un carácter cualitativo y descriptivo, pues a partir de técnicas como entrevistas, estudios de caso y observaciones, se logró describir las características del SB. Freudenberger (como se citó en Maslach et al., 2001) escribió en 1975 los primeros artículos sobre este fenómeno y lo denominó con el término coloquial burnout, empleado anteriormente para referirse al efecto del abuso crónico en el consumo de sustancias adictivas. En la segunda fase, en los años 80 , se estudió el SB de una forma más sistemática y cuantitativa, utilizando la metodología de encuesta en poblaciones más amplias, lo que permitió reunir en tres dimensiones las características del SB. Este abordaje fue acompañado por investigaciones complementarias sobre la relación entre el SB y la satisfacción laboral, el compromiso y las intenciones de abandono.

EI SB se ha estudiado mayoritariamente en el ámbito de la salud y de la educación, pues surge principalmente en profesiones en las cuales el núcleo del trabajo está dado en la relación entre la persona que brinda un servicio y la persona que lo recibe (Maslach \& Jackson, 1981; Maslach et al. 2001). Sin embargo, en los años 90 se extendió el estudio del SB a otras profesiones.

Otero-López, Santiago y Castro (2008) han investigado desde un enfoque integrador las causas del SB. Entre ellas, exploraron una causa no tan estudiada en este campo, como son los eventos de vida desfavorables que puede tener una persona y su incidencia en el SB. Los autores sostienen que si bien es conocida la multicausalidad del SB, múltiples investigaciones estudian las variables aisladas en detrimento del estudio del fenómeno como un todo. Investigaciones que relacionan el SB con los rasgos de la personalidad, en particular, la personalidad tipo A (Hernández \& Olmedo, 2004; Alarcon, Eschleman \& Bowling, 2009; Raya, Moriana \& Herruzo, 2010), son ejemplos de ello. Adicionalmente, han surgido estudios longitudinales sobre los efectos que el ámbito laboral tiene sobre los pensamientos y sentimientos de los individuos, así como también aquellos que estudian el efecto de las intervenciones paliativas del SB (Maslach et al., 2001).

Otras investigaciones (Ducharme, Knudsen \& Roman, 2007; Moreno-Jiménez, Garrosa, Rodríguez, Martínez \& Ferrer, 2009) indican que los docentes que experimentan el SB, a raíz de condiciones laborales y de factores personales, es posible que tengan intenciones de abandono. Este hallazgo, sumado al descenso del egreso de profesores de los centros de Formación Docente en los últimos años, afirmado por Errandonea (2012) en el Anuario Estadístico del Ministerio de Educación y Cultura (Uruguay), agudiza la situación educativa.

Si bien existe abundante evidencia que respalda la relación estrecha que existe entre las condiciones laborales, los factores organizacionales y ciertas características de la personalidad con el SB, no se cuenta con datos de cuánta incidencia tiene cada uno de estos aspectos en docentes de Ciclo Básico en Montevideo.

En este contexto, el presente estudio se plantea como objetivos, en una muestra de profesores del Ciclo Básico de la educación pública de Montevideo: (1) realizar una aproximación a la situación actual del profesorado uruguayo respecto al SB. (2) Examinar cuáles variables sociodemográficas intervienen como facilitadoras del SB. (3) Indagar sobre la magnitud de la relación entre diversas áreas de la vida laboral, la personalidad, los eventos de vida estresantes, las intenciones de abandono y el SB.

Un enfoque social acerca del estudio del SB, constituye para Maslach et al. (2001) una invaluable contribución al bienestar y salud de las personas. Además, el SB docente no sólo afecta a la persona que lo padece, sino también a la organización en la cual desempeña su trabajo y al proceso de aprendizaje de los alumnos (Maslach, Jackson \& Leiter, 2010). El docente es un agente de cambio que incide directamente en el aprendizaje del estudiante, sentido último de la educación. En esta línea, Byrne (1999), afirma que un docente que padece el SB, estará menos apto para dedicarse a su trabajo y preparar clases adecuadas, tendrá menos simpatía y tolerancia hacia los estudiantes. También a nivel organizacional se empobrecen las relaciones, aumenta el ausentismo y decae su actuación. Por ende, invertir en la salud del colectivo docente es invertir en los educandos, logrando un cambio positivo en la sociedad. 
Cabe destacar, que "de la misma forma que en la bibliografía de habla francesa se ha introducido el concepto de malaise enseignant, que ha venido traduciéndose al castellano como malestar docente, en la bibliografía anglosajona aparece el término burnout [...]" (Esteve, 1994, p. 57). Este último término ha sido traducido al castellano con al menos diecinueve denominaciones distintas, aunque algunas presentan grandes similitudes entre sí (Gil-Monte, 2003). Dado que aún no existe un acuerdo acerca de la traducción más acertada al castellano y que a excepción de algunos casos la mayoría de los países dejó el término sin traducir (Maslach et al., 2001), se conserva en el presente trabajo la denominación más difundida y más utilizada en investigaciones, que ha sido síndrome de burnout.

\section{Marco teórico}

\section{¿Qué es el síndrome de burnout?}

El SB constituye una respuesta al estrés laboral crónico cuando fallan las estrategias de afrontamiento del sujeto. Posee carácter de síndrome en la medida en que los síntomas que origina se agrupan de manera sistemática en las personas que lo padecen (Ramos, 1999, como se citó en González-Trijueque et al., 2012). Según Gil-Monte y Peiró (2007):

Esta respuesta ocurre con frecuencia en los profesionales de las organizaciones de servicios (médicos, maestros, funcionarios de prisiones, policías, trabajadores sociales, etc.) que trabajan en contacto directo con los usuarios de tales organizaciones (pacientes, alumnos, presos, indigentes, etc.), (p. 13).

Sin embargo, también se ha estudiado el SB fuera del ámbito laboral, es el caso de deportistas (Guterman, 2004).

Gil-Monte y Peiró (2007), luego de una exhaustiva revisión bibliográfica, han delimitado el concepto, diferenciándolo de otros como el tedio, la depresión, la ansiedad, la fatiga, la neurosis existencial y el desencanto. Si bien todos ellos son conceptos próximos, se advierte de no confundirlos con el SB, pues éste tiene una identidad propia. Múltiples investigaciones han demostrado ya, que el SB surge a partir de la interacción del individuo con su trabajo, a diferencia de la depresión, por ejemplo, que permea otras áreas de la vida personal (Maslach et al., 2001). Asimismo, se ha explorado la relación entre el SB y la depresión; si bien las personas más propensas a sufrir depresión son más vulnerables a padecer el SB (Maslach et al., 2001), se ha arribado a la conclusión que el SB conduce a síntomas depresivos pero no recíprocamente (Shin, Noh, Jang, Park \& Lee, 2013).

La definición de SB más aceptada y utilizada, surge de la factorización del cuestionario Maslach Burnout Inventory (MBI). Maslach y Jackson (1981) logran definir el SB a través de tres dimensiones: el agotamiento emocional, la despersonalización y la baja realización personal.

El agotamiento emocional es la principal manifestación del SB, pues denota la dimensión del estrés. Implica sentirse vaciado, consumido y exhausto. Sin embargo, este componente no revela la relación que tiene el individuo con su trabajo (Maslach et al., 2001; 2010).

La despersonalización se caracteriza por poner distancia a la persona con la que se trabaja, adoptando actitudes insensibles y frías con ella e ignorando sus cualidades que lo hacen un ser único. (Maslach et al., 2001; 2010).

La consideración de una baja realización personal en el rol, ocurre cuando el individuo siente que no puede contribuir más con la persona que recibe el servicio y se decepciona, no encontrando otras áreas de recompensa (Maslach et al., 2010).

En cuanto al desarrollo del SB, no existe un consenso. Una teoría plantea que el SB se desarrolla inicialmente con la despersonalización, lo que provoca bajos sentimientos de realización personal y finaliza con agotamiento emocional. Una segunda teoría sostiene que las tres dimensiones del SB se desarrollan simultáneamente pero de manera independiente. Un tercer modelo indica que el agotamiento emocional constituye la primera fase del SB, seguida por la despersonalización y luego, por bajos sentimientos de realización personal (Leiter \& Maslach, 1988; Maslach et al., 2001; Taris, Le Blanc, Schaufeli \& Schreurs, 2005).

\section{Antecedentes del síndrome de burnout: es- tresores y facilitadores}

Existen diversos modelos explicativos del $\mathrm{SB}$, algunos desarrollados desde la teoría sociocognitiva del yo, otros desarrollados a partir de las teorías del intercambio social y por último, los elaborados desde la teoría organizacional. La primera línea de investigación pone énfasis en los factores personales; la segunda, destaca las percepciones del sujeto respecto a las interacciones; y la tercera línea resalta las variables or- 
ganizacionales y del contexto laboral (Gil-Monte \& Peiró, 1999). Sin embargo, Gil-Monte y Peiró (2007) sostienen que se deben conjugar las tres teorías para poder brindar una explicación completa y satisfactoria sobre la etiología del SB.

En otro orden, existen dos perspectivas, la clínica y la psicosocial, que permiten entender al SB de maneras diferentes. La perspectiva clínica hace alusión al SB como un estado al que llega el sujeto a raíz del estrés laboral, mientras que la perspectiva psicosocial considera al SB como un proceso que se desarrolla a partir de la interacción entre el sujeto y su entorno laboral (Gil-Monte \& Peiró, 2007).

Bajo esta última perspectiva, los antecedentes pueden ser clasificados en estresores, que contribuyen a desencadenar el SB, y en facilitadores, que hacen que la persona sea más proclive a padecerlo.

Los estresores son principalmente factores vinculados al contexto laboral y a la esfera organizacional. En este sentido, Leiter y Maslach (2011) identificaron, luego de su revisión bibliográfica, seis áreas de la vida laboral del sujeto que pueden contribuir en el SB: carga de trabajo, control, recompensa, comunidad, justicia y valores. A partir de allí, ambos elaboraron un instrumento que permite medir dichos dominios, denominado Areas of Worklife Survey.

La sobrecarga laboral es uno de los predictores del SB más investigados. La misma está determinada por la excesiva demanda de trabajo, tanto en carácter cuantitativo (asociado a demasiadas tareas para cumplir) como cualitativo (asociado a tareas muy difíciles), es decir, implica hacer demasiado, con escaso tiempo y pocos recursos (French \& Caplan, 1973, como se citó en Cooper \& Marshall, 1976).

La falta de control en el trabajo es considerada un antecedente del SB, en la medida en que el trabajador no percibe tener la autonomía suficiente para tomar decisiones que le permitan resolver problemas y lograr la efectividad en su labor (Leiter \& Maslach, 2011).

La categoría recompensa, que incluye la recompensa monetaria, social e intrínseca, se refiere a la consistencia entre el reconocimiento percibido por el trabajador y sus expectativas (Leiter \& Maslach, 2011).

La dimensión comunidad comprende el entorno social de la organización. El relacionamiento con los compañeros de trabajo es fundamental. Argyris y Cooper (como se citó en Cooper \& Marshall, 1976) señalan que las bue- nas relaciones de trabajo constituyen el factor central en la salud individual y organizacional. En los ámbitos en donde existe un buen relacionamiento, se obtiene apoyo y ayuda de los compañeros de trabajo, en caso de ser necesarios, y se percibe la conformación de un equipo de trabajo, logrando alcanzar, de esta manera, el sentimiento de comunidad. De lo contrario, Leiter y Maslach (2011) advierten que los conflictos mal resueltos con compañeros de trabajo constituyen el factor más destructivo, pues generan sentimientos de frustración.

La revisión de la literatura permite afirmar, que un buen clima organizacional atenúa la aparición del SB. Éste existe si el ambiente de trabajo es relajado, confortable, alentador, solidario, comunicativo y sin desigualdades. Con el término justicia, Leiter y Maslach (2011) se refieren a este último aspecto, es decir, a la existencia de equidad en reglas y procedimientos.

El área valores comprende la existencia o no de compatibilidad entre las expectativas del trabajador y los objetivos de la organización. El conflicto entre valores personales y organizacionales tiene una incidencia clave en el nivel alcanzado en el SB del trabajador (Leiter \& Maslach, 2011).

Componen los facilitadores del SB algunas variables de carácter socio-demográfico, la personalidad, las estrategias utilizadas por el sujeto y los eventos con alto impacto en la vida de la persona.

Algunas variables sociodemográficas se han identificado como predictores del SB. El género femenino tiende a puntuar más alto en la dimensión agotamiento emocional mientras el género masculino tiende a tener más altas puntuaciones en la dimensión despersonalización. Las personas jóvenes, solteras y aquellas que no tienen hijos son las que obtienen valores más elevados en la escala del SB. En cuanto a la antigüedad en la profesión, particularmente en la docencia, existe mayor riesgo de contraer el SB cuánto menor experiencia posea el docente. (Cordes \& Dougherty, 1993; Gil-Monte \& Peiró, 2007; Maslach, 1993, en Maslach et al. 2010).

Respecto a la personalidad, según GilMonte y Peiró (2007):

Existe un cierto grado de acuerdo entre los investigadores que estudian el síndrome de quemarse por el trabajo al considerar que los profesionales más proclives a quemarse por el trabajo son sujetos empáticos, sensibles, humanos, con dedicación profesional, idealistas, altruistas, obsesivos, entusiastas y susceptibles de identificarse excesivamente con los demás (p. 73). 
Particularmente, múltiples investigaciones han encontrado relación entre el SB y el patrón de conducta tipo A (Costa \& Monteiro, 2002; Hernández \& Olmedo, 2004; Alarcon et al. 2009; Raya et al., 2010). Bortner (1969) ha diseñado un instrumento para medir este tipo de personalidad que si bien refleja unidimensionalidad, Edwards, Baglioni y Cooper (1990) identificaron tres dimensiones en él: la competitividad (comportamiento enfático), la impaciencia (urgencia en el tiempo) y la implicancia laboral (actividad polifásica). La competitividad se caracteriza por el deseo de sobresalir; la impaciencia, que es reconocido como el componente central, implica abarcar varias tareas a la vez, realizándolas lo antes posible, demostrando un sentido crónico de la urgencia; la implicancia laboral ocurre cuando la persona pone mucho énfasis en su trabajo buscando siempre el reconocimiento y la efectividad (Dorta, 2002). Respecto a este último aspecto, los resultados de la investigación de Brown y Roloff (2011) revelaron que los docentes que invierten tiempo extra al trabajo, son más propensos a padecer el SB.

Las personas con este tipo de personalidad, están en general muy comprometidas con su profesión pero no lo hacen de una manera sana: siempre están apresuradas, realizando varias tareas a la vez y proponiéndose metas continuamente sin disfrutar el proceso hasta conseguirlas (Dorta, 2002). Por ello, este tipo de personalidades son muy propensas al estrés. Friedman y Rosenman (como se citó en Edwards et al., 1990), quienes describieron por primera vez el patrón de conducta tipo A en el año 1959, notaron además que estas características se encuentran mayoritariamente en personas con enfermedades cardiovasculares.

En cuanto a las estrategias de afrontamiento, Lazarus y Folkman (como se citó en Blanch, Aluja \& Biscarri, 2002) las definen como esfuerzos cognitivos y conductuales en constante cambio, desarrolladas para manejar demandas específicas internas o externas que desbordan los propios recursos del individuo; las clasifican en aquellas orientadas al problema, que intentan actuar directamente sobre la situación que provoca estrés, y aquellas emocionales que intentan moderar la respuesta propia ante eventos estresantes. Si bien las primeras son más efectivas, Blanch et al. (2002) sostienen que en el contexto de los servicios sociales, conviene combinar ambas estrategias para lograr una mayor adaptación y bienestar psicológico, dado que los profesionales no cuentan con suficiente capacidad de control sobre los estresores del entorno. Un excesivo afrontamiento al problema, cuando existen pocas posibilidades de controlar, sería contraproducente para el sujeto, tornándolo más proclive a padecer el SB. En este sentido, Kareaga (2004) afirma que la relajación que trasciende el plano fisiológico, facilitando los recursos internos relacionados con la inteligencia emocional, cumple un papel central en el afrontamiento, aún en el marco de una intervención organizacional.

Otero-López et al. (2008) han encontrado relación entre el SB y las experiencias de vida estresantes reunidas en el Life Events Inventory (LEI), diseñado por Hammen y Mayol en 1982. Según el estudio de Spurgeon, Jackson y Beach (2001), los diez eventos más estresantes en la vida de una persona, en orden decreciente de severidad, son los siguientes: fallecimiento del cónyugue, sentencia de prisión, fallecimiento de un familiar directo, intento de suicidio de un familiar directo, endeudamiento, período sin hogar, seria enfermedad en un familiar directo, desempleo del jefe de familia, divorcio y desintegración de la familia. La vivencia de estas crisis tienen un alto impacto en la vida del trabajador, dejándolo más vulnerable y facilitando la aparición del SB.

\section{Consecuencias del síndrome de burnout}

Desde la perspectiva psicosocial se entiende que el SB trae aparejado diversas consecuencias tanto para el sujeto como para la organización. Dichas consecuencias podrían agruparse en cinco niveles o planos distintos: fisiológico, emocional, interpersonal, actitudinal y conductual (Kahill, 1988, como se citó en Cordes \& Dougherty, 1993).

El nivel fisiológico incluye, por ejemplo, la aparición de fatiga, insomnio, alergias e infecciones, y desórdenes gastrointestinales. A nivel emocional se sufre, por ejemplo, una baja autoestima, irritabilidad, ansiedad, soledad, impotencia y depresión. En el plano interpersonal se puede observar una socialización disminuida hacia los compañeros de trabajo y personas a las cuales se les brinda el servicio. En cuanto a lo actitudinal, se manifiesta insatisfacción, apatía y un menor compromiso laboral, entre otros. Las consecuencias conductuales implican, por ejemplo, la reducción de la cantidad de trabajo, el deterioro de la calidad del servicio, el absentismo laboral, la rotación organizacional y 
la propensión al abandono. La sintomatología percibida en los dos primeros planos, repercute directamente en la salud del sujeto, mientras que los síntomas percibidos en los demás planos afectan la relación sujeto-organización, así como también las relaciones interpersonales del sujeto en el ámbito privado. (Cordes \& Dougherty, 1993; Maslach et al., 2001; Gil-Monte \& Peiró, 2007).

\section{Método}

\section{Participantes}

De la investigación participaron 279 profesores, de una población total de $4.732^{2}$, que estaban ejerciendo la docencia directa en Ciclo Básico, en calidad de efectivos o interinos ${ }^{3}$, en algún liceo público ${ }^{4}$ de Montevideo al momento de la aplicación del cuestionario, en el año 2013.

La selección de centros donde se llevó a cabo el estudio se realizó mediante un muestreo aleatorio estratificado con el objetivo de obtener una participación con composición lo más heterogénea posible y se acotó el estudio a los campos Montevideo, Ciclo Básico y Educación Pública, con el propósito de permitir su viabilidad.

En primer lugar, con la ayuda de la Inspección de Institutos y Liceos, se confeccionó un listado de liceos públicos de Ciclo Básico de Montevideo con ciertas características para la conformación del marco muestral. Se solicitaron liceos con dos turnos diurnos con el objetivo de encuestar docentes en el turno matutino y vespertino, pues el alumnado es diferente en ambos turnos, pudiendo interferir esto en el los niveles de SB de los docentes. Se exigió la aplicación del plan Reformulación 2006, para que los docentes trabajaran bajo los mismos reglamentos y programas y éstos no intervinieran produciendo distintos niveles del SB. Excluir a los liceos "aluvión", permitió suponer que el nivel socioeconómico del barrio donde se ubicaba el centro educativo era el mismo que

2 El dato se obtuvo a partir del total de horas de clase pagas en el presupuesto de julio de 2013, comunicadas por el Departamento de Computación de la División Hacienda (Consejo de Educación Secundaria, Administración Nacional de Educación Pública). Cabe mencionar, que no se tuvo en cuenta en la población total a los docentes en calidad de suplente.

3 El docente efectivo es el titular del cargo y el interino es aquel que se desempeña en un cargo sin titular durante todo el año lectivo.

4 En Uruguay, se denomina liceo público a todo centro estatal de enseñanza secundaria de ambos ciclos (Ciclo Básico y Bachillerato) en la modalidad general (no tecnológica). el nivel socioeconómico de su alumnado. No se incluyeron los liceos diurnos con alumnado adulto y extraedad, ni se encuestaron docentes del turno nocturno, con el objetivo de asegurar que todos los participantes dictaran clase en al menos un grupo con alumnado adolescente, pues éstos suelen ocasionar más problemas de disciplina, lo que se traduce en mayor estrés para el docente, según las investigaciones de Salanova, Martínez y Lorente (2005) y Marqués, Lima, y Lopes (2005).

De lo anterior se desprende, que el universo lo constituyen los centros educativos públicos de Montevideo de Ciclo Básico, con dos turnos diurnos, que tienen el plan Reformulación 2006, que no son liceos "aluvión" 5 y que no tienen alumnado adulto ni extraedad. Las unidades de análisis son los docentes interinos o efectivos que dictan clase en dichos centros.

En segundo lugar, se agruparon los barrios de Montevideo en 11 categorías según el puntaje detallado Índice de Nivel Socioeconómico (INSE) completo (Llambí \& Piñeyro, 2012) que tenían asociado que toma valores entre 0 y 10. Luego, se distribuyeron los centros educativos, que constituyen el universo de este estudio, a partir del puntaje INSE de su barrio (Apéndice). De allí, se eligió un liceo al azar de cada estrato, con el fin de agrupar a los docentes en dichos liceos y facilitar el proceso de encuesta. Cabe mencionar, que si bien el total de estratos fue de 11, conformaron la muestra 10 liceos, pues no se encontró ningún centro con las características mencionadas en el párrafo anterior que estuviera ubicado en los barrios que poseen el máximo puntaje INSE.

La muestra de docentes se compuso por un censo de los docentes correspondientes a los centros educativos seleccionados en la muestra. Ésta estuvo compuesta predominantemente por el género femenino, 214 mujeres $(76,7 \%)$. De los 279 docentes encuestados, 142 de ellos tenían una edad entre 30 y 40 años (50,9\%), 143 profesores estaban casados (51,3\%) y 155 tenían hijos (55,6\%). Asimismo, 214 docentes poseían título de grado (76,7\%), 233 contaban con formación docente ${ }^{6}(83,5 \%), 178$ trabajaban en más de un liceo $(63,8 \%), 201$ trabajaban

5 Se suele denominar liceo "aluvión" a aquel que es capaz de absorber poblaciones estudiantiles de distintas zonas de Montevideo (incluso lejanas a su ubicación), por su estratégica ubicación geográfica y su buena locomoción. Se caracteriza por un alumnado con nivel socioeconómico muy heterogéneo.

6 Se consideró dentro de esta categoría a los profesores que declararon estar cursando o haber cursado algún año en un centro de Formación Docente al momento de la investigación. 
más de 20 horas reloj semanales (72,0\%) y 114 poseían una experiencia laboral de 10 años o menos en la profesión $(40,9 \%)$.

\section{Procedimiento}

Se visitó a cada centro en horario de coordinación docente y se le entregó a cada profesor el cuestionario, acompañado de una carta que expresaba el propósito de la investigación y solicitaba la cumplimentación del cuestionario de forma anónima y voluntaria. Las personas entregaron en forma escrita su consentimiento informado a participar de la investigación. Cada docente utilizó el tiempo de la coordinación para completar el cuestionario de forma individual y devolverlo al investigador. A partir del mismo se recabaron datos sociodemográficos del docente, aspectos sobre el SB y sus antecedentes, así como también se indagó sobre las intenciones de abandono. Los datos fueron analizados con el programa estadístico informático Statistical Package for the Social Sciences (SPSS, 2011).

\section{Variables e Instrumentos}

- Síndrome de Burnout. Para medir esta variable, se eligió como instrumento el Maslach Burnout Inventory-Educators Survey (MBI-ES) (Maslach, Jackson \& Schwab, 1986) dada su gran aceptación internacional. Este instrumento consta de 22 ítems distribuidos en las tres dimensiones que integran el SB. El agotamiento emocional, es evaluado en el MBI-ES a través de 9 ítems (ítem de ejemplo: "Me siento fatigado cuando me levanto por la mañana y tengo que enfrentarme con otro día de trabajo"). La despersonalización comprende en el MBI-ES 5 ítems (ítem de ejemplo: "Creo que trato a algunos estudiantes como si fuesen objetos impersonales"). La falta de realización personal es evaluada en el MBI-ES a través de 8 ítems (ítem de ejemplo: "Creo que estoy influyendo positivamente con mi trabajo en la vida de los demás").

Cada sentimiento es evaluado con una escala de frecuencia tipo Likert en la cual "0" corresponde a "Nunca" y " 6 " corresponde a "Diariamente".

Altos puntajes en las dimensiones agotamiento emocional y despersonalización, así como, bajos puntajes en realización personal revelan presencia del SB (Maslach et al., 2010).
- Vida laboral. Para medir esta variable se seleccionó el instrumento Areas of Worklife Survey -AWS- (Leiter \& Maslach, 2000), que presenta 18 ítems en su versión más corta. Éstos se evalúan con una escala Likert con 5 puntos de anclaje en la cual "1" corresponde a "Totalmente en desacuerdo" y "5" corresponde a "Totalmente de acuerdo".

Cada una de las seis áreas es evaluada con tres ítems: sobrecarga laboral (ítem de ejemplo: "No tengo tiempo para hacer el trabajo que debería ser hecho"), control (ítem de ejemplo: "Tengo influencia en las decisiones que afectan mi trabajo"), recompensa (ítem de ejemplo: "Recibo reconocimiento de otros por mi trabajo"), comunidad (ítem de ejemplo: "Los miembros de mi grupo de trabajo cooperan unos con otros"), justicia (ítem de ejemplo: "Las oportunidades son decididas sólo por mérito"), valores (ítem de ejemplo: "Mis objetivos profesionales personales son consistentes con los objetivos que la organización se establece").

Tal como mencionan Leiter y Maslach (2011) en el manual del instrumento, un promedio mayor o igual a 2,5 en cada dimensión indica una alta congruencia entre las expectativas y valores, en este caso, de la institución educativa y los del docente.

- Personalidad. Para medir la variable personalidad se aplicó la Escala de Bortner (EB) diseñada en 1969. Este instrumento presenta 14 ítems, en cada uno de los cuales figuran 2 frases o adjetivos que representan comportamientos opuestos, uno de ellos define el patrón de conducta tipo $\mathrm{A}$ y el otro al patrón de conducta tipo B.

La competitividad es evaluada en la EB a través de 6 ítems (ítem de ejemplo: "Satisfecho con el trabajo-Ambicioso "), la impaciencia comprende 4 ítems (ítem de ejemplo: "Puedo esperar pacientemente-Impaciente cuando espero") y la implicancia laboral es valorada a través de 4 ítems (ítem de ejemplo: "Muchos intereses-Pocos intereses fuera del trabajo"). Altos puntajes indican personalidad tipo $\mathrm{A}$.

- Eventos de vida. Procurando evaluar la variable eventos de vida, se incluyó en el cuestionario un apartado con una adaptación del instrumento LEI. En el mismo figuraban los diez eventos más estresantes señalados por Spurgeon et al. (2001), de forma que el participante pudiera marcar aquellos que había

7 Los rasgos de la personalidad tipo A están escritos en letra Italic en el texto 
experimentado en el último año. Además, tenía la opción de indicar algún otro evento vivido que considerara relevante.

De acuerdo a la investigación realizada por Spurgeon et al. (2001), quienes actualizaron los pesos originales desarrollados en el LEI por Cochrane y Robertson (1973), se asignaron pesos de severidad entre 0 y 100 a cada evento de vida que le hubiera ocurrido al encuestado y luego se sumaron para obtener el valor de la variable.

- Intenciones de abandono. Ajzen (1985) plantea en su teoría del comportamiento planificado que las intenciones que tienen los individuos son buenos determinantes de su conducta, pues es esperado que las personas actúen de acuerdo a sus intenciones. Este es el motivo por el cual la propensión al abandono se suele medir a través de las intenciones de abandono.

En este sentido, Gálvez (2007, como se citó en Martínez, Moreno \& Ferrer, 2009) diseñó un cuestionario con cuatro preguntas para medir las intenciones de abandono en el profesorado universitario (ítem de ejemplo: "He tenido deseos de abandonar la profesión universitaria"). A partir de este cuestionario, se construyó un instrumento para medir las intenciones de abandono (IA), adaptando las preguntas para esta investigación y añadiendo opciones para el caso de respuesta afirmativa con el fin de discriminar entre un abandono parcial (e. g. reducir las horas) o un abandono total de la profesión. Se otorgaron pesos a las diferentes opciones de respuesta en cada ítem y luego se sumaron para obtener el valor de la variable. Puntajes elevados indican una mayor intención de abandono.

\section{Variables sociodemográficas}

Se incluyeron en el cuestionario preguntas para recabar datos acerca de los siguientes aspectos sociodemográficos: edad, género, estado civil, número de hijos, antigüedad docente, formación académica, cantidad de lugares y horas de trabajo.

\section{Modelo de análisis}

La Figura 1 representa el modelo de análisis del presente estudio. El mismo fue construido a partir de la revisión bibliográfica de la literatura conceptual y de investigación sobre el SB.

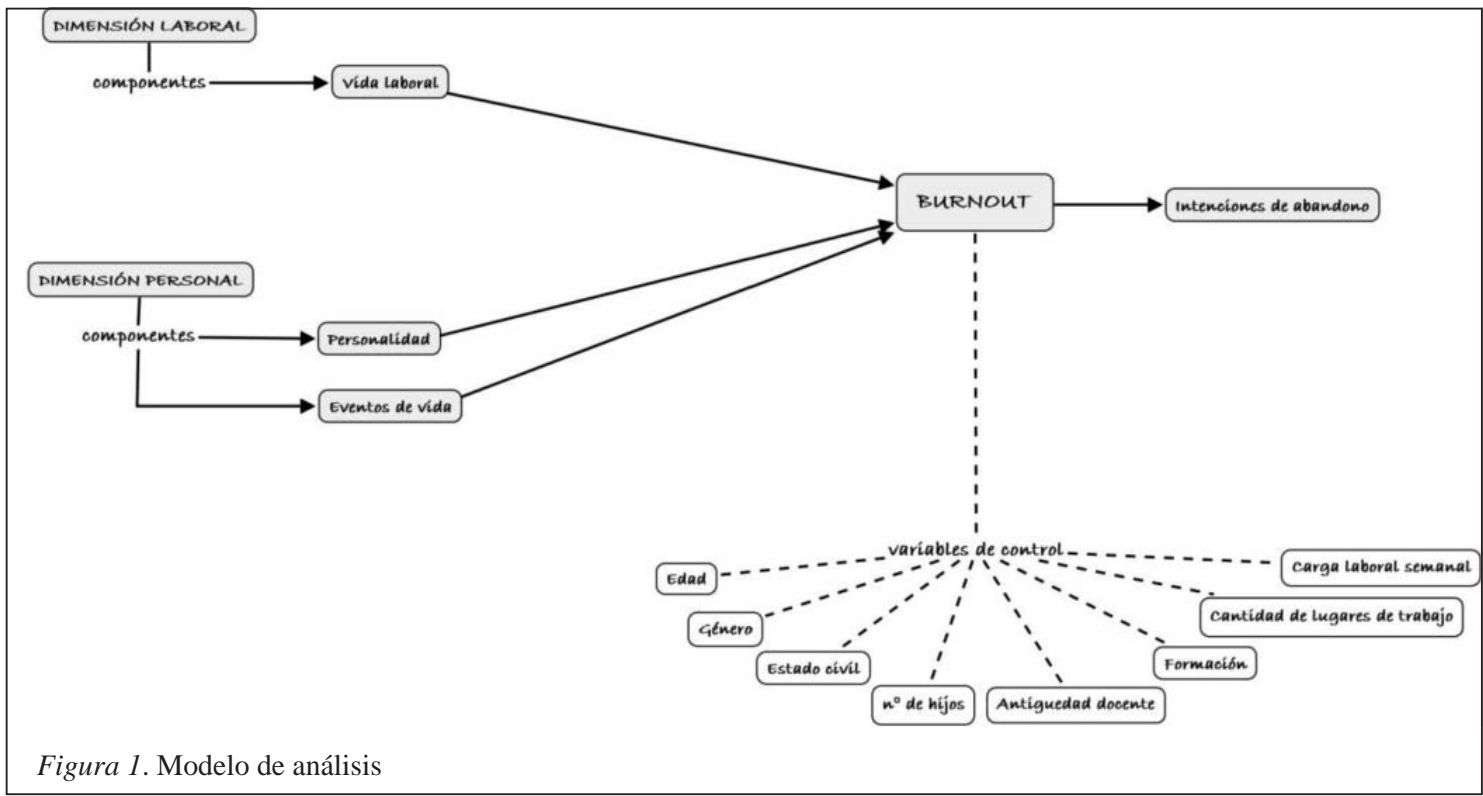




\section{Resultados}

Una primera aproximación descriptiva se recoge en la Tablas 1 y 2, que muestran las medias y las desviaciones típicas en las subescalas del MBI-ES y el análisis porcentual de los resultados obtenidos en cuanto a las dimensiones del SB respectivamente.

Tabla 1

Medias y desviaciones estándar para las subescalas del MBI-ES

\begin{tabular}{lcccc}
\hline \multicolumn{1}{c}{ Dimensiones del SB } & $\begin{array}{c}\text { Casos } \\
\text { Válidos }\end{array}$ & $\begin{array}{c}\text { Rango de } \\
\text { Puntaje }\end{array}$ & Media & $\begin{array}{c}\text { Desviación } \\
\text { Estándar }\end{array}$ \\
\hline Agotamiento emocional & 260 & $0-54$ & 21,38 & 11,28 \\
Despersonalización & 266 & $0-30$ & 4,61 & 4,65 \\
Baja realización personal & 256 & $0-40$ & 36,25 & 7,24 \\
\hline
\end{tabular}

Tabla 2

Distribución de los resultados obtenidos en las subescalas del SB (en porcentajes)

\begin{tabular}{lccc}
\hline $\begin{array}{l}\text { Nivel en } \\
\text { el SB }\end{array}$ & $\begin{array}{c}\text { Agotamiento } \\
\text { Emocional }\end{array}$ & Despersonalización & $\begin{array}{c}\text { Realización } \\
\text { Personal }\end{array}$ \\
\hline Bajo & 35,4 & 84,2 & 53,5 \\
Moderado & 36,2 & 10,2 & 25,4 \\
Alto & 28,5 & 5,6 & 21,1 \\
Total & 100,0 & 100,0 & 100,0 \\
\hline
\end{tabular}

Los índices que más destacan son los de agotamiento emocional y realización personal, mientras que la despersonalización alcanza bajos puntajes. Casi un $30 \%$ de los profesores encuestados indica encontrarse agotado emocionalmente, resultado que coincide con el reportado por los maestros montevideanos en la Encuesta UNESCO de Condiciones de Trabajo y Salud Docente realizada en 2004 que fue de 29\% (como se citó en Robalino \& Körner, 2005; Cornejo, 2008). Asimismo, se puede observar que la quinta parte del profesorado presenta sentimientos de baja realización personal y un porcentaje reducido de los docentes experimenta despersonalización.

Un 4,2\% del profesorado posee niveles elevados en el SB (agotamiento emocional y despersonalización altos, realización personal baja) y un $42 \%$ de los docentes presentan una o más dimensiones del SB, pudiendo esto indicar que se trata de una población en riesgo de padecerlo.

Si comparamos las medias y las desviaciones estándar de las dimensiones del SB con las recopiladas por Vercambre, Brosselin, Gilbert, Nerrière y Kovess-Masféty (2009), luego de una revisión bibliográfica de estudios sobre el SB en profesores de distintos países de Europa (Francia, Holanda, Grecia, Italia, España, Alemania, Finlandia, Reino Unido y Bélgica) se encuentran varias similitudes. Al igual que en el presente estudio, en la mayoría de los países, la media del agotamiento emocional es aproximadamente de 20 puntos con una desviación de 10 puntos; tanto la media como la desviación estándar de la despersonalización toman un valor promedio de 4 puntos; la realización personal asciende a valores en torno a los 30 puntos con una desviación de 8 o 9 puntos.

Sin embargo, existen más diferencias que similitudes con los resultados alcanzados en las investigaciones llevadas a cabo por Díaz Bambula, López Sánchez y Varela Arévalo (2012) en Colombia y por Santana, De Marchi, Junior, Girondoli y Chiappeta (2012). Por un lado, en Colombia no se ha encontrado dentro de la muestra, docentes con las características del SB alto y los porcentajes de profesores con bajo SB es superior en todas las dimensiones al alcanzado en este estudio. Por otro lado, los resultados en Brasil, muestran una media de despersonalización bastante superior a la alcanzada por los profesores montevideanos.

Para establecer la relación entre las dimensiones del SB con las variables sociodemográficas, se realizó un análisis de datos categóricos a través de tablas de contingencia, a partir de las cuales se analizó el estadístico Chi-cuadrado de Pearson para poder determinar la independencia entre las variables contrastadas. De este modo, la Tabla 3 indica que la dimensión agotamiento emocional se asocia significativamente con la variable género, siendo la población femenina la que más padece agotamiento emocional. Este hallazgo es consistente con la bibliografía (Cordes y Dougherty, 1993; Maslach, 1993, citado en Maslach et al. 2010). La misma también sostiene que el género masculino tiene, por lo general, puntuaciones más altas en la dimensión despersonalización y una realización personal disminuida, aunque no se ha encontrado relación entre dichas variables en este estudio.

Si bien más de un $60 \%$ de las personas que presentan altos índices en el SB tienen entre 25 y 40 años en este estudio, la prueba de Chicuadrado no ha encontrado relación entre ambas variables. Sin embargo, la literatura afirma que son los jóvenes los que más padecen SB, particularmente los valores más altos en el SB se encuentran en personas de dicho rango de 
edad (Farber, 1984, como se citó en Gil-Monte \& Peiró, 2007).

La prueba de Chi-cuadrado no ha revelado relación entre el estado civil y el SB en el presente estudio, aunque se ha investigado que las personas casadas puntúan más bajo en el SB que las no casadas, al igual que las personas con hijos (Cordes \& Dougherty, 1993; Gil-Monte \& Peiró, 2007; Maslach, 1993, citado en Maslach et al. 2010).

Si se considera el grado de formación en cuanto a la educación recibida por la persona, no existe ninguna relación con las dimensiones del SB. En cambio, si clasificamos a las personas según si tienen formación docente o no, la prueba de Chi-cuadrado revela dependencia de la dimensión realización personal respecto a esta variable sociodemográfica ya que las personas con formación docente demostraron tener más altos sentimientos de realización personal que aquellas personas cuya formación de origen es distinta a la docencia.

La prueba de Chi-cuadrado también revela dependencia de la dimensión realización personal respecto a las variables antigüedad y cantidad de lugares de trabajo. El porcentaje de docentes que poseen altos sentimientos de realización personal es relativamente mayor en las personas que tienen una antigüedad de 20 años o más en la profesión, hallazgo que se encuentra alineado con lo que plantean GilMonte y Peiró (2007) al afirmar que existe un riesgo mayor de padecer SB cuando la persona tiene menos experiencia. Los docentes que trabajan en tres o más lugares también tienen una realización personal aumentada.

Asimismo, el agotamiento emocional se encuentra relacionado con la variable carga laboral semanal, en la medida en que los docentes que demuestran altos niveles de agotamiento

Tabla 3

Pruebas Chi-cuadrado derivadas de las tablas de contingencia Dimensiones del SB*Variables sociodemográficas

\begin{tabular}{|c|c|c|c|c|}
\hline & $\begin{array}{l}\text { Casos } \\
\text { Válidos }\end{array}$ & $\begin{array}{c}\text { Chi-cuadrado } \\
\text { de Pearson }\end{array}$ & GI & $\begin{array}{l}\text { Sig. Asintótica } \\
\text { (bilateral) }\end{array}$ \\
\hline Agotamiento Emocional*Edad & 252 & 3,767 & 4 & 0,438 \\
\hline Agotamiento Emocional*Género & 260 & 9,808 & 4 & $0,044^{\star}$ \\
\hline Agotamiento Emocional*Estado civil & 260 & 5,113 & 8 & 0,745 \\
\hline Agotamiento Emocional ${ }^{\star}$ Número de hijos & 260 & 9,212 & 8 & 0,325 \\
\hline Agotamiento Emocional*Antigüedad docente & 253 & 2,205 & 6 & 0,900 \\
\hline Agotamiento Emocional*Formación & 260 & 5,037 & 8 & 0,754 \\
\hline Agotamiento EmocionalFormación docente & 260 & 1,123 & 4 & 0,891 \\
\hline Agotamiento Emocional*Cantidad de lugares de trabajo & 248 & 2,643 & 4 & 0,619 \\
\hline Agotamiento Emocional*Carga laboral semanal & 249 & 14,642 & 6 & $0,023^{\star}$ \\
\hline DespersonalizaciónEdad & 258 & 5,216 & 4 & 0,266 \\
\hline DespersonalizaciónGénero & 266 & 1,635 & 4 & 0,803 \\
\hline Despersonalización`Estado civil & 266 & 7,014 & 8 & 0,535 \\
\hline Despersonalización`Número de hijos & 266 & 2,741 & 8 & 0,950 \\
\hline Despersonalización`Antigüedad docente & 258 & 3,992 & 6 & 0,678 \\
\hline Despersonalización`Formación & 266 & 7,374 & 8 & 0,497 \\
\hline Despersonalización*Formación docente & 266 & 1,768 & 4 & 0,778 \\
\hline Despersonalización ${ }^{*}$ Cantidad de lugares de trabajo & 251 & 0,735 & 4 & 0,947 \\
\hline Despersonalización ${ }^{\star}$ Carga laboral semanal & 252 & 7,492 & 6 & 0,278 \\
\hline Realización Personal*Edad & 248 & 4,587 & 4 & 0,332 \\
\hline Realización Personal*Género & 256 & 2,926 & 4 & 0,570 \\
\hline Realización PersonalEstado civil & 256 & 6,926 & 8 & 0,545 \\
\hline Realización Personal*Número de hijos & 256 & 8,565 & 8 & 0,380 \\
\hline Realización Personal*Antigüedad docente & 248 & 13,692 & 6 & $0,033^{\star}$ \\
\hline Realización Personal*Formación & 256 & 6,346 & 8 & 0,608 \\
\hline Realización Personal`Formación docente & 256 & 10,449 & 4 & $0,034^{\star}$ \\
\hline Realización Personal*Cantidad de lugares de trabajo & 242 & 10,329 & 4 & $0,035^{\star}$ \\
\hline Realización Personal*Carga laboral semanal & 243 & 2,780 & 6 & 0,836 \\
\hline
\end{tabular}


emocional son los que tienen mayor cantidad de horas de trabajo semanales.

Si consideramos el contexto en el que trabajan los docentes, la prueba de independencia de Chi-cuadrado constata relación entre éste y las dimensiones del SB. Mientras que el $7 \%$ de los docentes que trabajan en los contextos con nivel socioeconómico más alto poseen elevados niveles en el SB, este porcentaje asciende al $17 \%$ en los contextos más bajos.

Para presentar los resultados de los demás instrumentos se confeccionó la Tabla 4 que indica las medias y desviaciones estándar de las escalas y subescalas de los mismos.

Tabla 4

Medias y desviaciones estándar para las escalas y subescalas de los instrumentos

\begin{tabular}{lcccc}
\hline \multicolumn{1}{c}{ Instrumento } & $\begin{array}{c}\text { Casos } \\
\text { Válidos }\end{array}$ & $\begin{array}{c}\text { Rango de } \\
\text { Puntaje }\end{array}$ & Media & $\begin{array}{c}\text { Desviación } \\
\text { Estándar }\end{array}$ \\
\hline AWS & 229 & $1-5$ & 3,28 & 0,51 \\
Carga de trabajo & 257 & $1-5$ & 3,00 & 0,88 \\
Control & 261 & $1-5$ & 3,38 & 0,81 \\
Recompensa & 266 & $1-5$ & 3,35 & 0,78 \\
Comunidad & 265 & $1-5$ & 3,56 & 0,87 \\
Justicia & 259 & $1-5$ & 3,04 & 0,77 \\
Valores & 270 & $1-5$ & 3,33 & 0,68 \\
EB & 251 & $0-168$ & 94,50 & 16,15 \\
Competitividad & 259 & $0-72$ & 35,49 & 7,79 \\
Impaciencia & 269 & $0-48$ & 29,80 & 8,01 \\
Implicancia Laboral & 269 & $0-48$ & 28,94 & 6,16 \\
LEI & 278 & $0-930$ & 81,77 & 109,10 \\
IA & 255 & $0-10$ & 3,60 & 2,49 \\
\hline
\end{tabular}

Se ha constado que el mayor grado de ajuste en la vida laboral del docente está relacionado, en este caso, con las áreas comunidad, control, recompensa y valores. La congruencia es significativamente menor en las áreas carga de trabajo y justicia.

El $71,3 \%$ de los docentes presenta personalidad tipo A. Sin embargo, al observar las dimensiones de esta variable, se puede apreciar que tanto la impaciencia como la implicancia laboral, características de este tipo de personalidad, están presentes en los docentes encuestados, pero no así la competitividad.

El $49,6 \%$ de los docentes no ha padecido, en el entorno de un año, ninguno de los 10 eventos de vida registrados en el cuestionario, mientras que un $26,3 \%$ manifiesta un alto puntaje en este instrumento.

Si se analizan las intenciones de abandono, el $60,4 \%$ de los docentes presenta entre un nivel moderado y alto.

Al explorar la matriz de correlaciones, presentada en la Tabla 5 se puede apreciar que todas las variables de estudio que mostraron correlaciones significativas, lo han hecho en la dirección esperada.

Si se analiza el grado de intensidad de las mismas (sin considerar aquellas entre una variable y alguna de sus dimensiones), la más fuerte se observa entre dos dimensiones del área laboral, pues la recompensa por el trabajo correlaciona significativa y positivamente con el control del trabajo. En este orden, cabe destacar también la correlación significativa y positiva entre las áreas recompensa y comunidad, las áreas justicia y control, y las áreas valores y justicia.

Al igual que en otras investigaciones (Raya et al., 2010; Costa \& Monteiro, 2002) la personalidad tipo A se asocia con un coeficiente significativo y positivo con el SB. Entre las dimensiones de la personalidad tipo A, las que presentan una correlación más intensa son la impaciencia y la implicancia laboral que están asociadas de forma significativa y positiva.

Tabla 5

Correlaciones entre los factores de primer y segundo orden de los cuestionarios

\begin{tabular}{|c|c|c|c|c|c|c|c|c|c|c|c|c|c|c|c|c|}
\hline & 1 & 2 & 3 & 4 & 5 & 6 & 7 & 8 & 9 & 10 & 11 & 12 & 13 & 14 & 15 & 16 \\
\hline \multicolumn{17}{|l|}{ 1. Agotamiento Emocional } \\
\hline 2. Despersonalización & $0,47^{* *}$ & & & & & & & & & & & & & & & \\
\hline 3. Realización Personal & $-0,29^{* *}$ & $-0,33^{* *}$ & & & & & & & & & & & & & & \\
\hline 4. SB & $0,74^{* *}$ & $0,65^{* *}$ & $-0,67^{* *}$ & & & & & & & & & & & & & \\
\hline 5. Carga de trabajo & $-0,45^{* *}$ & $-0,25^{* *}$ & $0,15^{*}$ & $-0,33^{* *}$ & & & & & & & & & & & & \\
\hline 6. Control & $-0,21^{* *}$ & $-0,14^{*}$ & $0,19^{* *}$ & $-0,21^{* *}$ & $0,33^{* *}$ & & & & & & & & & & & \\
\hline 7. Recompensa & $-0,38^{* *}$ & $-0,19^{* *}$ & $0,31^{* *}$ & $-0,36^{* *}$ & $0,39 *$ & $0,51^{* *}$ & & & & & & & & & & \\
\hline 8. Comunidad & $-0,14^{*}$ & $-0,14^{*}$ & $0,19^{* *}$ & $-0,17^{* *}$ & 0,08 & $0,23^{* *}$ & $0,40^{\star \star}$ & & & & & & & & & \\
\hline 9. Justicia & $-0,04$ & $-0,05$ & 0,03 & $-0,06$ & $0,17^{* *}$ & $0,42^{* *}$ & $0,27^{\star \star}$ & $0,17^{\star \star}$ & & & & & & & & \\
\hline 10. Valores & $-0,03$ & $-0,07$ & $0,13^{*}$ & $-0,12$ & $0,13^{*}$ & $0,34^{* *}$ & $0,38^{\star *}$ & $0,30^{* *}$ & $0,44^{\star *}$ & & & & & & & \\
\hline 11. Área Laboral & $-0,33^{* *}$ & $-0,24^{* *}$ & $0,25^{* *}$ & $-0,32^{* *}$ & $0,57^{* *}$ & $0,72^{* *}$ & $0,74^{\star \star}$ & $0,60^{\text {** }}$ & $0,62^{\star *}$ & $0,63^{\star \star}$ & & & & & & \\
\hline 12. Competitividad & 0,10 & 0,05 & $-0,01$ & 0,08 & $-0,14^{*}$ & $-0,12$ & $-0,13^{\star}$ & $-0,14^{*}$ & $-0,18^{* *}$ & $-0,17^{\star \star \star}$ & $-0,24^{\star \star}$ & & & & & \\
\hline 13. Impaciencia & $0,28 * *$ & 0,09 & $-0,18^{* *}$ & $0,26 * *$ & $-0,22 * *$ & $-0,12$ & $-0,09$ & $-0,10$ & $-0,01$ & 0,02 & $-0,13$ & $0,34^{\star \star}$ & & & & \\
\hline 14. Implicancia Laboral & $0,16^{*}$ & 0,08 & $-0,06$ & 0,11 & $-0,14^{*}$ & $-0,08$ & $-0,06$ & 0,01 & $-0,03$ & 0,01 & $-0,05$ & $0,17^{\star \star}$ & $0,41^{\star \star}$ & & & \\
\hline 15. Personalidad & $0,26 * *$ & 0,09 & $-0,12$ & $0,20 * *$ & $-0,24^{* *}$ & $-0,13^{*}$ & $-0,13^{\star}$ & $-0,10$ & $-0,08$ & $-0,07$ & $-0,18^{\star \star}$ & $0,71^{\star \star}$ & $0,82^{\star \star}$ & $0,66^{\star \star}$ & & \\
\hline 16. Eventos de vida & $0,21^{* *}$ & $0,14^{*}$ & $-0,00$ & $0,18^{* *}$ & $-0,10$ & 0,06 & $-0,03$ & 0,03 & 0,12 & 0,09 & 0,03 & $-0,02$ & $-0,08$ & 0,07 & $-0,02$ & \\
\hline 17. Intenciones de abandono & $0,47^{* *}$ & $0,27^{* *}$ & $-0,27^{* *}$ & $0,46^{* *}$ & $-0,28^{* *}$ & $-0,20^{* *}$ & $-0,29^{\star *}$ & $-0,12$ & $-0,11$ & $-0,15^{\star}$ & $-0,30^{* *}$ & $-0,00$ & 0,06 & 0,10 & 0,09 & $0,22^{\star \star}$ \\
\hline
\end{tabular}


Respecto a las dimensiones del SB, se destaca la correlación significativa y positiva entre el agotamiento emocional y la despersonalización. La primera de ellas también correlaciona significativa pero negativamente con la carga de trabajo, hallazgo que se comparte con la investigación de Lee y Ashforth (1996).

Considerando las relaciones entre las intenciones de abandono y el SB, se aprecia que ambas correlacionan de forma positiva y significativa. El índice de correlación asciende ligeramente cuando sólo se considera el factor agotamiento emocional; lo mismo ocurre en la investigación de Zhang y Zhang (2012). En este sentido, la OIT (1981, como se citó en Esteve, 1994) afirma que este factor parece constituir una razón esencial de los abandonos que se observan en la profesión docente. Este resultado coincide completamente con las conclusiones de las investigaciones de Moreno-Jiménez et al. (2009) y de Ducharme et al. (2007).

\section{Discusión y conclusiones}

El presente trabajo de investigación se ha centrado en el estudio del SB en una muestra de 279 profesores de enseñanza secundaria de la educación pública en Montevideo. En este trabajo se ha estudiado el SB como variable criterio; a las áreas de la vida laboral como estresores; a las variables sociodemográficas, la personalidad y los eventos de vida como facilitadores; $y$ finalmente, a las intenciones de abandono como una de las variables consecuentes del SB.

El principal objetivo de esta investigación ha sido realizar una aproximación a la situación que enfrenta nuestro país actualmente, en particular Montevideo, respecto al SB docente. En este sentido, se observó que un $4,2 \%$ de los docentes encuestados padecen el SB y un $42 \%$ se encuentra en situación de riesgo. Al considerar sus dimensiones, se destacan los valores alcanzados en el agotamiento emocional y la realización personal, no así en la despersonalización. Este último dato es positivo para la sociedad pues es la dimensión que más trae consecuencias sobre el estudiante, ya que el endurecimiento emocional del docente trae aparejado un trato distante hacia a sus alumnos.

Sin embargo, cabe señalar que el porcentaje de personas que solicita licencias médicas es más alto entre las que padecen altos niveles de agotamiento emocional en la escala del SB (Robalino \& Körner, 2005) y esto podría constituir un sesgo en la muestra, ya que dichas personas no fueron consideradas para integrar la misma, lo que podría impedir obtener un porcentaje más elevado en esta dimensión así como también en la escala del SB.

Se ha puesto de manifiesto que algunas variables sociodemográficas se presentan como facilitadoras del SB, tal como la teoría lo predice. El género femenino y docentes con elevada cantidad de horas semanales de trabajo tienden a puntuar más alto en la dimensión agotamiento emocional. Asimismo, los docentes con poca antigüedad en la profesión, que no tienen formación docente y que trabajan hasta en dos centros educativos son propensos a manifestar una baja realización personal.

De esta investigación se desprende que juegan un papel importante en este proceso la personalidad, los eventos de vida y la congruencia lograda en el área laboral, hallazgos que se encuentran alineados con un conjunto de investigaciones sobre el tema. Las personas con personalidad tipo A, caracterizadas por la impaciencia, la implicancia laboral y la competitividad, así como también las personas que han vivido eventos de vida estresantes en el entorno de un año tienden a experimentar mayores niveles en el SB. En cambio, la congruencia en el área laboral (particularmente en cuanto al control de trabajo, la carga laboral, la recompensa, la comunidad, la justicia y los valores) y el SB correlacionan de forma significativa e inversa. Dicha variable antecesora presenta un mayor índice de correlación con el SB respecto a las variables de carácter personal.

El contexto del lugar de trabajo también presentó asociación con los niveles alcanzados por el docente en el SB, siendo los porcentajes de docentes con SB más altos en los contextos socioeconómicos críticos.

Se pudo constatar además, que las intenciones de abandono aumentan de forma significativa cuando los docentes padecen el SB.

No obstante, los resultados presentados deben ser analizados con cautela, dado que esta muestra no necesariamente es representativa y los autoinformes no necesariamente reflejan las vivencias de las personas.

Por otro lado, si bien se aplicaron los cuestionarios a los docentes en el menor tiempo posible, dentro del mismo tramo del año lectivo y alejado de los períodos de vacaciones, se debe tener en cuenta que en el año lectivo en el que se recabaron los datos, surgieron más paros 
docentes que en otros años. Este hecho pudo alterar los valores obtenidos en la medida en que la discontinuidad de clases pudo haber causado una disminución del agotamiento emocional y de la despersonalización, así como también, la insatisfacción con las condiciones laborales manifestada en los paros pudo haber afectado la realización personal.

Otra limitación de este estudio ha sido operativizar un constructo tan complejo como la personalidad sólo en dos categorías, lo que responde sólo a efectos prácticos, pues permite la comparación con otras investigaciones.

Sería pertinente en el futuro realizar un análisis con regresión múltiple para conocer los niveles de contribución de las variables predictoras al pronóstico del SB.

Sin embargo, tal y como señala la literatura sobre el tema, los resultados obtenidos en esta investigación -teniendo en cuenta las limitaciones presentadas- aportan datos de interés en la medida en que dejan en evidencia la necesidad de intervenir a nivel institucional para prevenir la presencia del SB en el profesorado. En este sentido, la investigación de Blanch et al. (2002) sostiene que invertir esfuerzos por lograr un aumento en la realización personal del profesorado tiene mayores efectos positivos que actuar sobre las otras dos dimensiones del SB, por lo que, en primer lugar, sería conveniente que la sociedad revalorice la profesión docente, pues la percepción generalizada entre los profesores es que existe una escasa valoración social del trabajo docente (Robalino \& Körner, 2005).

Lo que afirma Esteve (1994) también se aplica a nuestra situación: "Es injusto que nuestra sociedad nos considere los únicos responsables de los fracasos de un sistema escolar masificado, apresuradamente maquillado para hacer frente a la avalancha de la crisis social, económica e intelectual de nuestra sociedad." (p.19). Se considera imprescindible pues, adoptar un enfoque proactivo en cuanto a la prevención (GonzálezTrijueque et al., 2012), aunando esfuerzos por restablecer el equilibrio entre el docente y su vida laboral para no hacer de la educación, como advierte Esteve (1994), una profesión imposible.

\section{Referencias}

Ajzen, I. (1985). From intentions to actions: A theory of planned behavior. Heidelberg: Springer.

Alarcon, G., Eschleman, K. J., \& Bowling, N. A. (2009). Relationships between personality variables and burnout: $A$ meta-analysis. Work \& stress, 23(3), 244-263.
Blanch, A., Aluja, A., \& Biscarri, J. (2002). Síndrome de quemarse en el trabajo (burnout) y estrategias de afrontamiento: un modelo de relaciones estructurales. Revista de psicología del trabajo y de las organizaciones, 18(1), 57-74.

Bortner, R. W. (1969). A short rating scale as a potential measure of pattern A behavior. Journal of chronic diseases, 22(2), 87-91.

Brown, L. A., \& Roloff, M. E. (2011). Extra-Role Time, Burnout, and Commitment: The Power of Promises Kept. Business Communication Quarterly, 74(4), 450-474.

Byrne, B. M. (1999). The nomological network of teacher burnout: A literature review and empirically validated model. En Vandenberghe R. y Huberman A.M. (eds.). Understanding and preventing teacher burnout. A sourcebook of international research and practice. Cambridge: Cambridge University Press.

Cochrane, R., \& Robertson, A. (1973). The life events inventory: a measure of the relative severity of psycho-social stressors. Journal of Psychosomatic Research, 17(2), 135-139.

Cooper, C. L., \& Marshall, J. (1976). Occupational sources of stress: A review of the literature relating to coronary heart disease and mental ill health. Journal of occupational psychology, 49(1), 11-28.

Cordes, C. L., \& Dougherty, T. W. (1993). A review and an integration of research on job burnout. Academy of management review, 18(4), 621-656.

Cornejo, R. (2008). Entre el sufrimiento individual y los sentidos colectivos: salud laboral docente y condiciones de trabajo. Docencia, 35, 77-85.

Costa, W., \& Monteiro, A. M. (2002). A influência de fatores de personalidade e de organização do trabalho no burnout em profissionais de saúde. Estudos de Psicologia, 19(1), 37-48.

Díaz Bambula, F., López Sánchez, A. M., \& Varela Arévalo, M. T. (2012). Factores asociados al síndrome de burnout en docentes de colegios de la ciudad de Cali, Colombia. Universitas Psychologica, 11(1), 217-227.

Dorta, M. R. (2002). Validez del constructo patrón de conducta tipo A. Departamento de Personalidad, Evolución y Tratamientos Psicológicos, Universidad de la Laguna.

Ducharme, L. J., Knudsen, H. K., \& Roman, P. M. (2007). Emotional exhaustion and turnover intention in human service occupations: The protective role of coworker support. Sociological Spectrum, 28(1), 81-104.

Edwards, J. R., Baglioni Jr. A.J., \& Cooper, C. L. (1990). The psychometric properties of the Bortner type A scale. British journal of Psychology, 81(3), 315-333.

Errandonea, G. (2012). Anuario Estadístico de Educación. Resumen ejecutivo 2011. Montevideo: Ministerio de Educación y Cultura (MEC). Recuperado de http:// educacion.mec.gub.uy/innovaportal/file/29425/1/resumen_ejecutivo.pdf

Esteve, J. M. (1994). El malestar docente. Barcelona: Paidós Ibérica.

Gil-Monte, P. (2003). Burnout syndrome: ¿síndrome de quemarse por el trabajo, desgaste profesional, estrés laboral o enfermedad de Tomás? Revista de Psicología del Trabajo y de las Organizaciones, 19(2), 181-197.

Gil-Monte, P. R., \& Peiró, J. M. (1999). Perspectivas teóricas y modelos interpretativos para el estudio del síndrome de quemarse por el trabajo. Anales de psicología, 15(2), 261-268.

Gil-Monte, P., \& Peiró, J.M. (2007). Desgaste psíquico en el trabajo: El síndrome de quemarse. Madrid: Síntesis. 
González-Trijueque, D., Giachero, S., \& Delgado, S. (2012). Riesgos psicosociales en el lugar de trabajo: aproximación teórica y marco legal en Uruguay. Ciencias Psicológicas, 6(1), 75-87.

Guterman, T. (2004). Burnout en deportistas. Lecturas: Educación física y deportes, 10(74). Recuperado de

http://www.efdeportes.com/efd74/burnout.htm

Hernández, G. L., \& Olmedo E. (2004). Un estudio correlacional acerca del síndrome del "estar quemado" (burnout) y su relación con la personalidad. Apuntes de psicología, 22(1), 121-136.

IBM Corporation (2011). IBM SPSS Statistics for Windows (20). Armonk, NY: IBM Corp.

Kareaga, A. (2004). Afrontamiento del estrés en las organizaciones: un programa de manejo a nivel individual/ grupal. Revista de Psicología del Trabajo y de las Organizaciones, 20(1), 77-93.

Lee, R. T., \& Ashforth, B. E. (1996). A meta-analytic examination of the correlates of the three dimensions of job burnout. Journal of applied Psychology, 81(2), 123-133.

Leiter, M. P., \& Maslach, C. (1988). The impact of interpersonal environment on burnout and organizational commitment. Journal of organizational behavior, 9(4), 297-308.

Leiter, M. P., \& Maslach, C. (2000). Areas of Worklife Survey. California: Mind Garden, Inc.

Leiter, M. P., \& Maslach, C. (2011). Areas of Worklife Survey Manual ( $5^{\text {th }}$ ed.). California: Mind Garden, Inc.

Llambí C., \& Piñeyro L. (2012). Índice de Nivel Socioeconómico (INSE). Montevideo: Cámara de Empresas de Investigación Social y de Mercado del Uruguay (CEISMU). Recuperado de http://www.gruporadar. com.uy/01/wp-content/uploads/2012/04/Informe-Nuevo-INSE-2011.pdf

Marqués, A., Lima, M. L., \& Lopes, A. (2005). Fuentes de estrés, burnout y estrategias de coping en profesores portugueses. Revista de Psicología del Trabajo y de las Organizaciones, 21(1-2), 125-143.

Martínez, M., Moreno, B. y Ferrer, R. (2009). Calidad de vida del profesorado universitario: el desgaste profesional. (Propuesta metodológica y resultados). Acciones e investigaciones sociales, 27, 131-169.

Maslach, C., \& Jackson, S. E. (1981). The measurement of experienced burnout. Journal of Organizational Behavior, 2(2), 99-113.

Maslach, C., Jackson S.E., \& Leiter M.P. (2010). Maslach Burnout Inventory Manual ( $3^{r d}$ ed.). California: Mind Garden, Inc.

Maslach, C., Jackson S.E., \& Schwab R.L. (1986). Maslach Burnout Inventory-Educators Survey (MBI-ES). California: Mind Garden, Inc.

Maslach, C., Schaufeli, W. B., \& Leiter, M. P. (2001). Job burnout. Annual review of psychology, 52(1), 397-422.
Moreno-Jiménez, B., Garrosa, E., Rodríguez, R., Martínez, M., \& Ferrer, R. (2009). El Burnout del Profesorado Universitario y las Intenciones de Abandono: Un Estudio Multi-Muestra. Revista de Psicología del Trabajo y de las Organizaciones, 25(2), 149-163.

Otero-López, J. M., Santiago Mariño, M. J., \& Castro Bolaño, C. (2008). An integrating approach to the study of burnout in university professors. Psicothema, 20(4), 766-772.

Raya A.F., Moriana, J.A., \& Herruzo J. (2010). Relación entre el Síndrome de Burnout y el patrón de conducta tipo A en profesores. Ansiedad y estrés, 16(1), 61-70.

Robalino, M., \& Körner, A. (2005). Condiciones de trabajo y salud docente: estudios de casos en Argentina, Chile, Ecuador, México, Perú y Uruguay. Santiago de Chile: Oficina Regional de Educación de la UNESCO para América Latina y el Caribe (OREALC / UNESCO). Recuperado de http://unesdoc.unesco.org/ images/0014/001425/142551s.pdf

Rossi, J. (2009) Profesión docente. Profesión cuestionada. Educarnos, 2(6), 1-60.

Salanova, M., Martínez, I. M., \& Lorente, L. (2005). ¿Cómo se relacionan los obstáculos y facilitadores organizacionales con el burnout docente?: Un estudio longitudinal. Revista de Psicología del Trabajo y de las Organizaciones, 21(1-2), 37-54.

Santana, Â. M. C., De Marchi, D., Junior, L. C., Girondoli, Y. M., \& Chiappeta, A. (2012). Burnout syndrome, working conditions, and health: a reality among public high school teachers in Brazil. Work: A Journal of Prevention, Assessment and Rehabilitation, 41, 3709-3717.

Shin, H., Noh, H., Jang, Y., Park, Y. M., \& Lee, S. M. (2013). A longitudinal examination of the relationship between teacher burnout and depression. Journal of Employment Counseling, 50(3), 124-137.

Spurgeon, A., Jackson, C. A., \& Beach, J. R. (2001). The Life Events Inventory: re-scaling based on an occupational sample. Occupational Medicine, 51(4), 287-293.

Taris, T. W., Le Blanc, P. M., Schaufeli, W. B., \& Schreurs, P. J. (2005). Are there causal relationships between the dimensions of the Maslach Burnout Inventory? A review and two longitudinal tests. Work \& Stress, 19(3), 238-255.

United Nations Organization for Education, Science and Culture [UNESCO], (2010). Datos mundiales de educación VII Ed. 2010/11. Recuperado de http://www.ibe.unesco. org/fileadmin/user_upload/Publications/WDE/2010/ pdf-versions/Uruguay.pdf

Vercambre, M. N., Brosselin, P., Gilbert, F., Nerrière, E., \& Kovess-Masféty, V. (2009). Individual and contextual covariates of burnout: a cross-sectional nationwide study of French teachers. BMC Public Health, 9(1), 1-12.

Zhang, J., \& Zhang, Q. (2012). Teacher Burnout and Turnover Intention in a Chinese Sample: The Mediating Role of Teacher Satisfaction. China Media Research, 8(2). 


\section{APÉNDICE}

Distribución de los liceos públicos de Ciclo Básico de Montevideo según el puntaje INSE de su barrio

\begin{tabular}{|c|c|c|}
\hline Puntaje INSE & Barrios de Montevideo & Liceos públicos de Ciclo Básico de Montevideo* \\
\hline 0 & Manga, Toledo Chico, Casavalle, La Paloma, Tomkinson & $\mathrm{N}^{\circ} 48, \mathrm{~N}^{\circ} 69, \mathrm{~N}^{\circ} 73$ \\
\hline 1 & $\begin{array}{l}\text { Bañados de Carrasco, Villa García, Manga Rural, Tres Ombúes, } \\
\text { Pbo. Victoria, Piedras Blancas, Las Acacias }\end{array}$ & $\mathrm{N}^{\circ} 25, \mathrm{~N}^{\circ} 39, \mathrm{~N}^{\circ} 52$ \\
\hline 2 & $\begin{array}{l}\text { Paso de La Arena, Manga, Conciliación, Casabó, Pajas } \\
\text { Blancas, Jardines del Hipódromo, Colón Centro y Noroeste, Pta. } \\
\text { Rieles, Bella Italia, Maroñas, P. Guarani, Lezica, Melilla, Castro, } \\
\text { Castellanos, Peñarol, Lavalleja, Ituzaingó, Flor de Maroñas, Villa } \\
\text { Muñoz, Retiro }\end{array}$ & $\begin{array}{l}N^{\circ} 24, N^{\circ} 40, N^{\circ} 43, N^{\circ} 44, N^{\circ} 45, N^{\circ} 49, N^{\circ} 50, \\
N^{\circ} 57, N^{\circ} 60, N^{\circ} 62, N^{\circ} 74\end{array}$ \\
\hline 3 & $\begin{array}{l}\text { Colón Sureste, Abayuba, Nuevo París, Villa Española, Las } \\
\text { Canteras, Cerro, Belvedere }\end{array}$ & $\mathrm{N}^{\circ} 11, \mathrm{~N}^{\circ} 51$ \\
\hline 4 & $\begin{array}{l}\text { Cerrito, Malvín Norte, Sayago, La Teja, Mercado Modelo y } \\
\text { Bolivar, Jacinto Vera, Reducto }\end{array}$ & $\mathrm{N}^{\circ} 22, \mathrm{~N}^{\circ} 23, \mathrm{~N}^{\circ} 41, \mathrm{~N}^{\circ} 42, \mathrm{~N}^{\circ} 47, \mathrm{~N}^{\circ} 70$ \\
\hline 5 & $\begin{array}{l}\text { Aires Puros, Unión, La Comercial, Figurita, Capurro, Bellavista, } \\
\text { Ciudad Vieja, Aguada, Brazo Oriental, Larrañaga, Paso de las } \\
\text { Duranas }\end{array}$ & $\mathrm{N}^{\circ} 21, \mathrm{~N}^{\circ} 37, \mathrm{~N}^{\circ} 53, \mathrm{~N}^{\circ} 55$ \\
\hline 6 & La Blanqueada, Cordón, Centro, Palermo, Atahualpa, Buceo & $\mathrm{N}^{\circ} 8, \mathrm{~N}^{\circ} 32$ \\
\hline 7 & $\begin{array}{l}\text { Prado, Nueva Savona, Barrio Sur, Pque. Batlle, V. Dolores, } \\
\text { Parque Rodó, Carrasco Norte }\end{array}$ & $\mathrm{N}^{\circ} 5, \mathrm{~N}^{\circ} 56, \mathrm{~N}^{\circ} 59$ \\
\hline 8 & Tres Cruces, Malvín & $\mathrm{N}^{\circ} 33$ \\
\hline 9 & Pocitos, Punta Gorda & $\mathrm{N}^{\circ} 7, \mathrm{~N}^{\circ} 20$ \\
\hline 10 & Carrasco, Punta Carretas & \\
\hline
\end{tabular}

*Nota: Se consideraron solo los liceos públicos de Ciclo Básico de Montevideo con dos turnos diurnos y con Reformulación 2006. No se incluyeron los liceos aluvión ni los liceos extraedad y de adultos.

Para citar este artículo:

Colino, N., \& Pérez de León, P. (2015). El síndrome de burnout en un grupo de profesores de enseñanza secundaria en Montevideo. Ciencias Psicológicas 9(1): 27 - 41 